\title{
Natural theory of relativity
}

\author{
Fayaz Tahir
}

Department of Civil Engineering, City College of the City University of New York, NY, USA

\section{Email address:}

fayaztahir313@hotmail.com

\section{To cite this article:}

Fayaz Tahir. Natural Theory of Relativity. American Journal of Astronomy and Astrophysics. Vol. 1, No. 3, 2013, pp. 23-40. doi: 10.11648/j.ajaa.20130103.11

\begin{abstract}
I have started this theory by deriving a different time dilation formula in an attempt to make the concepts of relativity more clear. I used the two postulates of special relativity i.e. the speed of light is constant for all inertial observers in free space i.e. vacuum and the same in all directions and the laws of physics are the same in all inertial frames together with the well-known fact that light takes a definite amount of time to travel between two points in space. I have then been able to get rid of the distortion, caused by the characteristics of the speed of light namely its constancy in all directions and the definite amount of time it takes to travel when it brings information from one point to another in space in combination with relative motion, in the form of infinite series terms. The distortion occurs symmetrically in the form of infinite series and leaves no skewness behind when got rid of. If we approximate the distorted value of a physical quantity to the first order, we get a distorted value. If we get rid of the distortion, in the form of infinite series, we get the actual value of the physical quantity. In the course of completing this theory I rejuvenated the concept of relative inertial kinetic energy and introduced relative gravitational acceleration at constant velocity in uniform circular motion. I have also been able to introduce the concept of gravitational shift in the dimensions of matter. My theory is very consistent.
\end{abstract}

Keywords: Characteristics of the Speed of Light, Relative Motion, Infinite Series, Distortion

\section{Introduction}

I always wondered why there is a bifurcation between the physics community one in favor of special relativity and the other against it. Then there came a moment in my life when I made up my mind to find the reason behind it. But just making up my mind was not the solution. I had to make attempts. And so I did. I started exploring different scenarios one after the other and after working continuously for three months or so I eventually hit the bull's eye. There was a moment on me when after making many apparently unsuccessful attempts I was going to give up the wild goose chase. I had the final equation in front of me but still no solution. Then all of a sudden the idea came to my mind and I took the limit and at once got what I was after; the relative inertial time dilation formula. And so I started my theory.

I have based my theory on two postulates. The first is that the speed of light is the speed of our information and constant for all inertial observers [1] and the second is, I believe, that the measurements taken in a frame of reference, of physical quantities of another frame of reference in relative motion, with the speed of our information as universal constant and requiring definite amount of time to travel between two relative inertial frames, are distorted symmetrically.
I have built my theory modestly and have not plunged into big results at once so that those who are familiar with special relativity may not stop reading my theory in the beginning. I have tried my best to give as much clarifications and derivations as possible.

I am well aware of the fact that my new interpretation of true relativity will not be easy to swallow for the old relativity guys. But I can give surety that it does not hurt in the least because I myself being an old relativity guy didn't have much difficulty in embracing the new interpretation of relativity. I believe that we should only consider that thought experiment to be true which resembles with what Lorentz transformations are advocating. Because I think results gotten by using Lorentz transformations should be held in more esteem than the thought experiments going contrary to the said transformations done by different researchers. My thought experiment that will come next is in perfect agreement with the inertial symmetry laid by Lorentz transformations.

\subsection{Lorentz Transformations - New Interpretation of Spacetime Dilation}

Before I proceed I deem it necessary to clarify in the very beginning that there are two facts about Lorentz transfor- 
mations. One of the facts is very clear and given in books on modern physics. But the other side of the picture of Lorentz transformations has not been given importance by many researchers. And the ignoring of that important fact, I believe, has been the apple of discord between the scientific community which has led to the bifurcation in the form of two groups; one in favor of special relativity and one against it. I will derive the one that has remained unimportant in the eyes of researchers.

The reader is expected to know the derivation of Lorentz transformations, before reading my interpretation, which can be had by reading any text on modern physics. So in order to explain, I have to redo the derivation of spacetime interval. The Lorentz transformations and Minkowski spacetime interval are as given below:

$$
\begin{gathered}
d X=\gamma(d x-v d t) \\
d Y=d y \\
d Z=d z \\
d T=\gamma\left(d t-v d x / c^{2}\right) \\
d S^{2}=-c^{2} d T^{2}+d X^{2}
\end{gathered}
$$

Inserting the values of $d X \& d T$ from the Lorentz transformations into the spacetime interval we proceed this time very cautiously as below:

$$
\begin{gathered}
d S^{2}=-c^{2} \gamma^{2}\left(d t-v d x / c^{2}\right)^{2}+\gamma^{2}(d x-v d t)^{2} \\
d S^{2}=\gamma^{2}\left(-c^{2} d t^{2}+d x^{2}+v^{2} d t^{2}-\frac{v^{2} d x^{2}}{c^{2}}\right)
\end{gathered}
$$

Now we know that for the constancy of the speed of light

$$
\frac{d x^{2}}{c^{2}}=d t^{2}
$$

Inserting the above expression into the interval and cancelling out the two terms we get

$$
\begin{gathered}
d S^{2}=\gamma^{2}\left(-c^{2} d t^{2}+d x^{2}\right) \\
d S^{2}=\gamma^{2} d s^{2}
\end{gathered}
$$

The above equation is the symmetric inertial spacetime dilation of the spacetime interval. Once again writing the above equation as below:

$$
\begin{gathered}
d S^{2}=\frac{-c^{2} d t^{2}+d x^{2}}{\left(1-\frac{v^{2}}{c^{2}}\right)} \\
d S^{2}=\frac{-c^{2} d t^{2}}{\left(1-\frac{v^{2}}{c^{2}}\right)}+\frac{d x^{2}}{\left(1-\frac{v^{2}}{c^{2}}\right)}
\end{gathered}
$$

We see that the only manner in which compatibility between the two inertial frames in relative motion can be maintained is through the following two natural and fundamental conditions as below which arise due to the con- stancy of the speed of light in both inertial frames:

$$
\begin{array}{lll}
d T^{2}=\frac{d t^{2}}{\left(1-\frac{v^{2}}{c^{2}}\right)} & \Rightarrow & T=\frac{t}{\sqrt{1-\frac{v^{2}}{c^{2}}}} \\
d X^{2}=\frac{d x^{2}}{\left(1-\frac{v^{2}}{c^{2}}\right)} \quad \Rightarrow & X=\frac{x}{\sqrt{1-\frac{v^{2}}{c^{2}}}}
\end{array}
$$

The above time and space dilation is in harmony with the constancy of the speed of light in both inertial frames i.e. $\frac{X}{T}=\frac{x}{t}=c$. The dilation of both space and time are built-in in the spacetime interval dilation. Moreover, the information of space is embedded in the information of time as will become clear later.

We see that the condition of compatibility between space and time of one frame with the other is met only when the warping of space and time of the inertial frame in relative motion is symmetric i.e. if time appears to have dilated then space also must appear to be the same and not vice versa. When I say symmetric warping of space and time, I mean that space and time are both dilated, instead of one being dilated and the other contracted, in order to maintain the constancy of the speed of light in vacuum. Another similar type of symmetric spacetime warping/dilation happens when electromagnetic signals bring information from one inertial frame to another in relative motion as will become clear next. By inertial I mean in deep space where Newton's first law of motion is valid i.e a body continues in its state of rest or of uniform motion (constant velocity and direction) in a straight line unless compelled by some external force to act otherwise.

\section{Simple thought Experiment -Relative Inertial Time Dilation}

Let us run a simple thought experiment. In order to determine the inertial time dilation of an inertial clock (clock whose functioning is unaffected by/or does not respond to changes in gravitational potential; we will make the gravitational correction later) at one point in space observed by an observer, having the same kind of inertial clock, in a relative inertial frame moving with constant velocity $v$ I have actually put two clocks (inertial) in the frame at rest relative to the moving frame, with a spatial distance $x$ apart. I will then calculate the time the moving observer measures of the two clocks according to his measurability with an inertial clock. The two equations describing the measurement of time of two different but synchronized inertial clocks in the rest frame will be scrutinized in the limiting case when the spatial distance $x$ between the two inertial clocks at rest tends to zero, hence making the two different times merge into one observation equal in magnitude. So, while the moving observer moves away from one inertial clock, it is simultaneously moving closer to the second. 


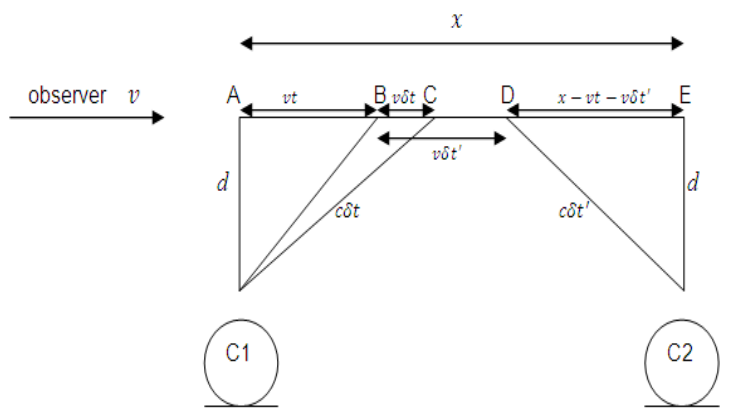

Figure 1. Relative inertial time dilation. Deriving the time interval $t$ of an inertial clock as a limit with the speed of our information in relative motion.

In the above Figure1 an observer is moving from the left to the right. Two inertial clocks $\mathrm{C} 1 \& \mathrm{C} 2$ synchronized with each other are placed $x$ distance apart. When the observer approaches right on top of the clock at a height of $d$, the inertial clocks $\mathrm{C} 1 \& \mathrm{C} 2$ start synchronically. After an elapse of time $t$, the observer reaches point B in its moving frame. Both inertial clocks at rest measure time $t$ as both are synchronized with each other in the rest inertial frame. Now, the observer tries to get the information of the time of $\mathrm{C} 1$ and by the time $\delta t$ the information at the speed of light (hereafter considered to be the speed of our information) reaches him he has reached point $\mathrm{C}$. Information speed takes definite amount of time (time light or light-like signals take to reach from $\mathrm{C} 1$ to observer at point $\mathrm{C}$ ). The observer at point $\mathrm{C}$ records this time to be $t_{1}$ according to the equation below:

$$
(v t+v \delta t)^{2}+d^{2}=(c \delta t)^{2}
$$

$\delta t=t_{1}-t \& c$ is the speed of our information

Where $\delta t$ is the definite amount of time in which the information reaches from $\mathrm{C} 1$ to observer at point $\mathrm{C}$. In the same manner we see that when the same observer gets the information of the time of $\mathrm{C} 2$ he has reached point $\mathrm{D}$. $\mathrm{D}$ does not necessarily need to be on the right of point $C$, it can be on the left of $\mathrm{C}$ depending upon the prevailing conditions between the observer and the inertial clocks. The same observer records the time of $\mathrm{C} 2$ according to the same power of measurability with which he measured the time of $\mathrm{C} 1$. He measures it $t_{2}$ given by equation 2 below:

$$
\begin{gathered}
\left(x-v t-v \delta t^{\prime}\right)^{2}+d^{2}=\left(c \delta t^{\prime}\right)^{2} \\
\delta t^{\prime}=t_{2}-t
\end{gathered}
$$

Where $\delta t^{\prime}$ is the definite amount of time in which the information reaches from $\mathrm{C} 2$ to observer at point D. Substituting the value of $d^{2}$ from equation 1 into 2, we get the following:

$$
\begin{gathered}
\left(x-v t_{2}\right)^{2}+(c \delta t)^{2}-\left(v t_{1}\right)^{2}=\left(c \delta t^{\prime}\right)^{2} \\
x^{2}-2 x v t_{2}+\left(c^{2}-v^{2}\right)\left(t_{1}^{2}-t_{2}^{2}\right)-2 c^{2} t\left(t_{1-} t_{2}\right)=0
\end{gathered}
$$

Now in the limiting case when $x$ tends to zero $t_{1} \& t_{2}$ merge to become $T$ as follows:

$$
T=\frac{t}{\left(1-v^{2} / c^{2}\right)}
$$

or

$$
T^{\text {ine }}=\frac{t^{\text {ine }}}{\left(1-v_{\text {ine }}^{2} / c^{2}\right)}
$$

Where the superscript "ine" stands for inertial and "gra" for gravitational.

The time $T^{\text {ine }}$ is independent of both $d$ and the observer going either to the left or to the right above the clock because all the three clocks are inertial and their rates of ticking are independent of gravitation. This is the equation for relative inertial time dilation. Inertial time is the time reckoned by an inertial clock (moving) with respect to another inertial clock (stationary) at rest in deep space where there is no gravity and Newton's first law of motion and its extension, done by natural relativity as will follow later, is applicable. The above thought experiment is done in deep inertial space i.e. no effects of gravitational acceleration or inertial acceleration.

If the above simple thought experiment is repeated with linear acceleration $a$ i.e. in a non-inertal frame using Newton's formalism of uniformly accelerated motion then the results would in no way be reflection of gravitational acceleration. We would end up in a cubic equation as below

$$
a^{2} T^{3}+3 u a T^{2}-2 c^{2}\left(1-\frac{u^{2}}{c^{2}}\right) T+2 c^{2} t=0
$$

Where $u$ is the initial instantaneous velocity of the moving observer when the stationary clocks started ticking time $t$. This is the equation for relative non-inertial time dilation. We see that the above equation reduces to equation 4 when there is no linear acceleration. The equation for relative gravitational time dilation will follow in Article 7.4.

If at any epoch of time my equation 4 above is tested in an experiment by using extremely precise time-reckoning devices or clocks then the experimentally recorded values $T^{\text {gra }}$ must be corrected by multiplying them with the factor of $f_{r}$ as will become clear later by reading Article 7.4. Moreover, when calculating $T$ with equation 4 above the factor $\beta^{2}=v_{\text {ine }}^{2} / c^{2}$ should also be replaced by $v_{g r a}^{2} / f_{r}^{2} c^{2}$ to make corrections for inertial time in the expression as below:

$$
T^{g r a} \times f_{r}=\frac{t^{g r a} \times f_{r}}{\left(1-v_{g r a}^{2} / f_{r}^{2} c^{2}\right)}=T^{\text {ine }}
$$

Gravitational clocks are at two different values of $r$, likewise the factor $f_{r}$ will be calculated for the gravitational clocks. With a little intuition we can easily derive the corresponding space dilation as follows. The distance measured with the speed of our information in the moving observer's 
frame of reference is $X=v T$. Putting the value of time $T$ from equation 4 above we get

$$
\begin{gathered}
X=v T \\
X=v \frac{t}{\left(1-v^{2} / c^{2}\right)} \\
X=\frac{v t}{\left(1-v^{2} / c^{2}\right)}
\end{gathered}
$$

Now $v t$ is equal to $x$ i.e. the distance travelled in the stationary frame of reference, therefore

$$
X^{i n e}=\frac{x^{i n e}}{\left(1-v_{i n e}^{2} / c^{2}\right)}
$$

Both observers one moving and one stationary measure each other's time and space as dilated in relative inertial motion. This property of relativity is called the reciprocal nature of inertial relativity or inertial frames of reference. We will see in Article 7.4 how this property of relativity breaks down in gravitational frames of reference when one gravitational clock in relative motion measures the time of another gravitational clock inertially. The two equations $4 \&$ $4 \mathrm{a}$ also guarantee the constancy of relative speed between the two frames i.e. $\frac{X}{T}=\frac{x}{t}=v$. This is the beauty of inertial relativity.

The relativistic effect of the measurement of time and space dilation as given by inertial relativity is temporary and reciprocal and therefore exists as long as relative inertial motion is maintained between the two bodies involved. But for the case of the two body relative gravitational motion between the Sun and Mercury or any other planet the relativistic effect is permanent but not reciprocal and hence supposed to have been in the observations made by Astronomers. Further clarifications of the previous statement will follow in Article 7.4.

The symmetry of spacetime dilation in equations $4 \& 4 \mathrm{a}$ is in perfect agreement with the new spacetime dilation interpretation of Lorentz transformations. Because I believe in Lorentz transformations [1], light (electromagnetic wave phenomenon) [2] warps space and time to keep its speed constant for all inertial observers and hence constant in all directions.

Furthermore, I believe, measuring the speed of light with space and time is not the same phenomenon as measuring space and time with the speed of light while in relative inertial motion. That is why we have relative inertial time and space dilation formulae. The space and time of frames of reference in relative inertial motion, I believe, seem to behave like a two dimensional pseudo-fabric which expands and contracts equally in both dimensions of space and time with an increase and decrease in relative speed between the two frames under investigation. Therefore, the warping of space and time i.e. the change in space and time of relative frames, while measuring them with the speed of light from a different frame in relative inertial motion is slightly different in magnitude but symmetrically the same like the Lorentz transformations' spacetime dilation. The effect of spacetime dilation is reciprocal i.e. both the observers in relative inertial motion measure, with the speed of our information, each other's spacetime as dilated. In addition, I also believe, that the information of space is remarkably built-in in the information of time.

I believe, what we feel with the speed of our information(s) in relative motion is not what we measure under the same conditions. We measure space dilation but we feel it as length contraction. This length contraction in the direction of motion is just due to the optical illusion and must never ever be made the basis and foundation for our intellectual thinking and calculations. The feeling of length contraction is just a mirage and a visual effect and has more to do with the third speed which is the speed of information that flows through the retina of the eye to the brain. This third speed interferes with the speed with which our retina is receiving information. The third-speed enigma, I believe, is the cause of the stubbornly persistent optical illusion of the shortening of length particularly in the direction of motion. Moreover, that illusion is independent of the relative velocity. In the presence of relative motion at Newtonian speeds it only gets aggravated slightly. I mean contraction is still more pronounced than new Lorentz space dilation at Newtonian speeds.

When we look at a long road of constant width in the direction of the length of the road, while standing stationary on the road, its width appears to be contracted the farther we look. It clearly means that the contracting of length has nothing to do with the measurements made of length in the direction of motion with the speed of our information from another frame with electronic instruments and not with eyes. Because of the involvement of our third speed for deciphering information through the retina and till the very end of the functioning of the brain, the brain outweighs the feel of space dilation by light in order to keep its speed constant in relative motion and hence causes the feeling of shortening of length by roughly the same amount as that third speed is smaller than the speed of light and the distance $d$ is greater between the observer and the length $x$, which is to be felt as follows:

$$
X_{f e l t}=\frac{f\left(v^{\prime}, d\right) x}{\sqrt{1-\frac{v^{2}}{c^{2}}}}
$$

When there is no relative motion $x$ can be considered in any direction. An approximate combination for the length contraction factor has been proposed as below:

$$
f\left(v^{\prime}, d\right)=\frac{\left(1-\mu \frac{v^{\prime}}{c}\right)}{\left(1-\mu \frac{v^{\prime}}{c}\left(1-\rho d e^{-\left\|v^{\prime}-c\right\|^{-1}}\right)\right)}
$$

Here $v^{\prime}$ is the third speed, $e$ is the Naperian logarithmic base and $\mu \& \rho$ are adjustable constants. 
On the contrary if, what we receive in the retina, we decipher in the brain with the same speed of light, i.e. the third speed becomes equal to the speed of light, till it reaches the very end of our brain functioning of psychological feeling, then we would be able to feel the exact dimensions of objects when there is no relative motion instead of a narrower road width as discussed before. But if, under the same conditions as just discussed above, we are moving with relative speed then we would be able to feel the space dilated according to new interpretation of Lorentz transformations. The difference of the two speeds of information i.e. one with which the information hits our retina and the second with which the information is deciphered in the brain till the very end of psychological state of human feeling, I think, is the root cause of the phenomenon.

The third speed, which I believe is small as compared to the mighty speed of light due to natural human physiological constraints, is the only obstacle to visually feel the new interpretation of Lorentz space dilation in relative motion.

Inserting the values of space and time dilation from equations $4 \& 4 \mathrm{a}$ into the Minkowski spacetime interval we have,

$$
\begin{gathered}
d S^{2}=-c^{2} d T^{2}+d X^{2} \\
d S^{2}=\frac{-c^{2} d t^{2}}{\left(1-\frac{v^{2}}{c^{2}}\right)^{2}}+\frac{d x^{2}}{\left(1-\frac{v^{2}}{c^{2}}\right)^{2}} \\
d S^{2}=\frac{d s^{2}}{\left(1-\frac{v^{2}}{c^{2}}\right)^{2}}
\end{gathered}
$$

\section{Analysis of Relative Inertial Time Dilation}

I believe there is no ideal frame of reference because our speed of information is finite and takes a definite amount of time to travel from one point to another in space. By ideal frame of reference I mean an inertial frame in which we can measure the change in space and time of other relative frames with an infinite speed of information. Due to lack of an ideal frame of reference, time becomes relative because its measurement made by different observers in different relative inertial frames moving with relative velocity becomes distorted with respect to the observers own frames of reference. This relative distortion in the measurement of time, space and eventually spacetime interval arises due to two parameters $v \& c$ competing each other in equation 4 , $4 \mathrm{a} \& 4 \mathrm{~b}$ respectively of the natural relativity, hereafter, called NR. Now let us analyze the equation for inertial time dilation given by NR as follows for relative velocities less than $c$ :

$$
\begin{gathered}
T=\frac{t}{\left(1-v^{2} / c^{2}\right)} \\
T=t\left(1-\frac{v^{2}}{c^{2}}\right)^{-1}
\end{gathered}
$$

$$
\begin{aligned}
& T=t\left(1+\frac{v^{2}}{c^{2}}+\frac{v^{4}}{c^{4}}+\frac{v^{6}}{c^{6}}+\frac{v^{8}}{c^{8}}+\cdots \infty\right) \\
& T=t+t\left(\frac{v^{2}}{c^{2}}+\frac{v^{4}}{c^{4}}+\frac{v^{6}}{c^{6}}+\frac{v^{8}}{c^{8}}+\cdots \infty\right)
\end{aligned}
$$

The most important term in the binomial series expansion on the right hand side of equation 6 is the first term of 1 . This represents the factor for the proper time and it remarkably retains the reality of the relative inertial frame in the scenario when there is no relative velocity in combination with the speed of our information. We can observe that the only thing responsible for distortion in our actual value of proper time $t$ in equation 7 above is the infinite series terms as below:

$$
\frac{v^{2}}{c^{2}}+\frac{v^{4}}{c^{4}}+\frac{v^{6}}{c^{6}}+\frac{v^{8}}{c^{8}}+\cdots \infty
$$

infinite series $\equiv$ distortion

So we can say that proper time and other physical quantities as well, as will come next, are distorted by relative motion in combination with the speed of light in the form of infinite series. Distortion is a relative effect measured in a relative frame and has nothing to do with the physical reality that lies within the frame of reference under investigation. One frame measures the other distortedly in relative motion. Basically when distortion i.e. infinite series, is got rid of then we are left with the actual picture of the frame of reference i.e. $T=t, X=x \& d S^{2}=d s^{2}$ as in equation $4,4 \mathrm{a} \& 4 \mathrm{~b}$ respectively. Distortion, as far as I think, is produced due to human incapability to measure with infinite speed of information in relative motion.

NR calculates the actual value of bending of ray of light near the Sun. For relative velocities far less than the speed of our information, the distorted values given by NR are pretty close to fixing the observed astronomical data, as in the case of the relativistic portion of the precession of the perihelion of Mercury, since the theory can easily approximate the infinite series to the first order. NR also gives the actual value of the relativistic precession of the perihelion of Mercury and other planets.

\section{Immeasurability}

According to NR, immeasurability, construed as human limited extent of measurability, is one of the causes of distortion. Immeasurability is due to the fact that our speed of information is finite, though huge, and it takes definite amount of time to reach from one point in one frame of reference to another point in another frame of reference. We can never receive information instantaneously without the intervention of time from one point in space to another. Immeasurability together with relative motion causes distortion.

The three things responsible for distortion are as follows:

1. Relative motion between two frames of reference.

2. Constancy of the speed of light in all directions.

3. Finite speed of light. 
The above three causes have woven the fabric of our distortion in the form of infinite series.

distortion $\equiv$ immeasurability + relative motion

\section{Relative Inertial Kinetic Energy- Distorted \& Actual}

The theory that I have developed incorporates the relative inertial frame, therefore everything will be considered in view of relative inertial frames of reference unless specified otherwise. We will take for granted the expression for the relativistic mass to be calculated in the same way as derived in [1], but this time with a slight modification in light of NR. Hence the expression for the relativistic mass that conserves momentum is as follows:

$$
\begin{array}{cc}
m(v)=\frac{m_{0}}{\left(1-v^{2} / c^{2}\right)} & \text { distorted } \\
m(v)=m_{0} & \text { actual }
\end{array}
$$

If we multiply equation 8 with $c{ }^{2}$ we have

$$
\begin{gathered}
m(v) c^{2}=\frac{m_{0}}{\left(1-v^{2} / c^{2}\right)} c^{2} \\
m(v) c^{2}=m_{0} c^{2}\left(1-v^{2} / c^{2}\right)^{-1} \\
m(v) c^{2}=m_{0} c^{2}\left(1+\frac{v^{2}}{c^{2}}+\frac{v^{4}}{c^{4}}+\frac{v^{6}}{c^{6}}+\frac{v^{8}}{c^{8}}+\cdots \infty\right) \\
m(v) c^{2}=m_{0} v^{2}+m_{0} c^{2}+m_{0} c^{2}\left(\frac{v^{4}}{c^{4}}+\frac{v^{6}}{c^{6}}+\frac{v^{8}}{c^{8}}+\cdots \infty\right) \\
m(v) c^{2}=m_{0} v^{2}+m_{0} c^{2}+\text { infinite series }
\end{gathered}
$$

We have seen from the above equation that the rest mass energy i.e. reserve energy, is distorted by the infinite series. So, after getting rid of distortions we have

$$
\begin{aligned}
& m(v) c^{2}=m_{0} c^{2}+m_{0} v^{2} \\
& E=m_{0} c^{2}+m_{0} v^{2} \\
& E=m_{0} c^{2}+m_{0} v^{2} \quad 0 \leq v \leq c
\end{aligned}
$$

Now here comes the mathematical trick. When we get rid of the distortion, we get rid of the binomial series approximating terms and when we get rid of the terms then we get rid of the condition as well. The condition associated with the binomial series terms is that the velocity should be much less than the speed of light. This peculiar condition is associated with the binomial series terms only. So it means that we can substitute the value of $c$ in place of $v$ in equation 14 above because we have got rid of the condition on velocity.

The above equation 14 is of remarkable importance and its full significance will be laid bare in Article 7.3 on the "Gravitational Shift in frequency of photons." We know that energies are of two main types i.e. kinetic and potential. Conjecturing in line with the same notion [1] we can conclude that equation 13 is more likely to be the expression for the total relative inertial kinetic energy of a body i.e. reserve energy and relative inertial kinetic energy. Equation 11 is the distorted picture of physical reality in relative inertial motion but equation 12 is the picture of the true nature of our frame of reference produced by NR.

Considering equation 13 above, we see that the total energy in the case of a photon travelling at $v=c$ turns out to be $2 m_{o} c^{2}$. According to NR, this is the total energy of a photon which includes the reserve energy and the kinetic energy. Now we see that the value of the kinetic energy of a photon is the same as its reserve energy. This observation, I believe, is the forerunner of a reasonable Principal of Equivalence of Energy as follows:

"The amount of energy that can be fed or supplied to matter is equivalent to the amount of energy that can be utilized by matter."

If we interpret the above principle the other way round then we can conclude that the maximum speed that matter can achieve is the speed of light. Matter is like a balloon made of elastic fabric and inflated to one half of the full mass of the air where it blows up. But as soon as it is inflated to the full mass of the air where it blows, it bursts and immediately loses everything, leaving behind nothing, not even the half of the air mass that it had before. The balloon fabric is like matter and the mass of air it contains is like energy. The unstable value of total energy ordinary matter can accommodate is therefore $2 m_{o} c^{2}$, which is also the stable value of total energy a photon can hold, giving the indication that for the ordinary matter to be stable at this value of total energy, the ordinary matter has to disintegrate into photons. Matter can travel with the speed of light but it will eventually convert into photons in view of NR. It clearly means that "energy is the smallest state of matter i.e. mass of a photon" in which matter can exist both as wave and matter, i.e. Einstein's declaration of duality of light [1], and does not convert into energy any further.

For the existence of matter in its ordinary physical form the following inequality for total energy $\mathrm{E}$ of matter holds true according to NR:

$$
m_{o} c^{2} \leq E \ll 2 m_{o} c^{2}
$$

I also believe that the only purpose of the peculiar matter (i.e. with duality) of photons is to warp space and time, firstly when maintaining constant speed for all inertial observers and secondly when taking/bringing information from one point to another in space in relative motion but differently in both scenarios.

In the light of NR ordinary matter cannot stay stable at the speed of light and therefore it has to disintegrate into photons. It concludes that photon is not the ordinary matter. Therefore the warping of space and time by the matter of photons does not give any indication of the warping of space and time, of the same order as photons, by ordinary matter like that of the Sun or planets. Matter has to be in motion and has to have a constant speed of $c$ in order to warp space and time appreciably. Therefore, I believe, ordinary physical matter is not meant for the warping of space and time. 
Nevertheless, we still have the possibility that even ordinary matter warps space and time but in an incredibly small portion but still sufficiently big enough for gravitational effects to take place smoothly.

Let us get back to where we left off i.e. immeasurability, together with relative motion, gives rise to distortion in measurement of the total relative inertial kinetic energy. Since the velocity of an object constitutes a moving frame of reference, therefore, when we measure its physical quantities, we get a distorted picture, due to relative motion, which has absolutely nothing to do with reality. Now we can measure the actual picture of physical quantities like energy, time and mass. Equation 13 is an example of the actual value of the total relative inertial kinetic energy of a body in relative inertial frame moving with relative velocity $v$.

Now let us try to extract the relative inertial kinetic energy of a body moving with zero linear acceleration and constituting an inertial frame of reference. Using the method as given in [1] we have

$$
\begin{aligned}
& \mathrm{KE}=\int F \mathrm{~d} s \\
& =\int \frac{\mathrm{d} p}{\mathrm{dt}} \mathrm{d} s \\
& =\int \frac{\mathrm{d} s}{\mathrm{dt}} \mathrm{d} p \\
& =\int v \mathrm{~d} p
\end{aligned}
$$

Now

$$
\begin{aligned}
p=m(v) v & =\frac{m_{0}}{\left(1-v^{2} / c^{2}\right)} v \\
\mathrm{KE} & =\int v \mathrm{~d} p
\end{aligned}
$$

Using integration by parts we have

$$
\begin{gathered}
\mathrm{KE}=v p-\int p \mathrm{~d} v+C \\
\mathrm{KE}=\frac{m_{0} v^{2}}{\left(1-v^{2} / c^{2}\right)}+C
\end{gathered}
$$

In the case of inertial motion with constant velocity $\mathrm{d} v=$ ty $\mathrm{d} v=0$ therefore the integral on the right above is zero. To calculate the value of the arbitrary constant of integration $\mathrm{C}$ we see that the relative inertial kinetic energy is zero at velocity equal to zero. Moreover, the arbitrariness of the scalar constant of integration suggests in itself any feasible value. Therefore the constant of integration is also zero. We obtain the results

$$
\mathrm{KE}=\frac{m_{0} v^{2}}{\left(1-v^{2} / c^{2}\right)}
$$

Now expanding the terms of the expression on the right above with binomial series we see

$$
\begin{aligned}
& \mathrm{KE}=m_{0} v^{2}\left(1+\frac{v^{2}}{c^{2}}+\frac{v^{4}}{c^{4}}+\frac{v^{6}}{c^{6}}+\cdots \infty\right) \\
& \mathrm{KE}=m_{0} v^{2}+m_{0} v^{2}\left(\frac{v^{2}}{c^{2}}+\frac{v^{4}}{c^{4}}+\frac{v^{6}}{c^{6}}+\cdots \infty\right)
\end{aligned}
$$

$$
\begin{gathered}
\mathrm{KE}=m_{0} v^{2}+\text { infinite series } \\
\mathrm{KE}=m_{0} v^{2} \text { actual }
\end{gathered}
$$

Equation 17 above gives the relative inertial kinetic energy according to NR. Newtonian kinetic energy formula $m_{0} v^{2} / 2$ by using Newton's formalism of uniformly accelerated non-inertial motion in a straight line and not gravitational motion in closed orbits is for comparatively large linear accelerations starting from zero velocity whereas the relative inertial kinetic energy formula $m_{0} v^{2}$ is for constant velocity and zero linear acceleration and meant for gravitational motion in bounded orbits. Since the planets have comparatively small linear accelerations therefore NR's relative inertial kinetic energy formula (which is basically Newton's gravitational planetary motion formula for circular orbital kinetic energy with eccentricity equal to zero as given by equation 31 ) is a better candidate for appreciably small linear accelerations as that of the planets revolving around the Sun than the Newtonian uniformly accelerated non-inertial and gravitational for unbounded parabolic orbit one.

Moreover, the Newton's uniformly accelerated motion kinetic energy formula happens to occur in the calculation of escape speed formula just by a coincidence for a body escaping gravity or going at infinite point in deep space away from matter. To add more, I believe, escape velocity does not make any bridge between relative gravitational planetary motion of bounded orbits and the relative inertial motion. But rather it is Newton's formula for uniform circular gravitational motion $\left(m v^{2}=m g r\right)$ which makes the bridge between inertial and gravitational motion. As an inference to what I have said above, Newton's formalism of uniformly accelerated motion in a straight line can depict planetary motion of bounded orbits weakly and cannot give approximate results. Adding more, it is a condition for escape speed that it must decrease with the distance from the gravitating matter till it vanishes at infinity and the escaping matter comes to a complete rest which is contrary to extension of Newton's first law of motion done by NR, as will come later, in which body stays at the final maximum velocity, in inertial motion, it had got from previous acceleration as soon as the force vanishes. Therefore the kinetic energy la $\left(m_{0} v^{2} / 2\right)$ is basically both a non-inertial and a gravitational formula for unbounded parabolic orbit and not at all an inertial one and therefore should have never ever been gotten by special theory of relativity if special theory of relativity talked about inertial motion at constant velocity.

When we compare the second term (relative inertial kinetic energy) on the right hand side of equation 13 with equation 17 , we see that it's the same and hence in agreement.

Let us analyze the picture of relativistic momentum [1] as follows:

$$
p=m(v) v=\frac{m_{0}}{\left(1-v^{2} / c^{2}\right)} v
$$


Now once again in line with NR the momentum of matter is given as follows, ignoring the terms of distortion:

$$
p=m_{0} v
$$

Equation 13 can also be written as

$$
E-p v=m_{0} c^{2}
$$

\section{Relative Gravitational Force and Acceleration - Distorted \& Actual}

For the calculation of relative non-inertial force, I used the same method [1] as I used in the calculation of relative inertial kinetic energy.

$$
\begin{gathered}
p=m(v) v=\frac{m_{0}}{\left(1-v^{2} / c^{2}\right)} v \\
F=\frac{\mathrm{d} p}{\mathrm{dt}}=\frac{\mathrm{d}}{\mathrm{dt}}\left(\frac{m_{0} v}{1-v^{2} / c^{2}}\right) \\
F=m_{0} \frac{\mathrm{d}}{\mathrm{dt}}\left(\frac{v}{1-v^{2} / c^{2}}\right) \\
F=m_{0} \frac{\mathrm{d}}{\mathrm{dv}}\left(\frac{v}{1-v^{2} / c^{2}}\right) \frac{\mathrm{d} v}{\mathrm{dt}}
\end{gathered}
$$

The above expression is equal to zero for inertial frame because $d v / d t=0$, but as soon as the change of frame from inertial to non-inertial happens we have

$$
F=m_{0} a \frac{\mathrm{d}}{\mathrm{dv}}\left(\frac{v}{1-v^{2} / c^{2}}\right)
$$

We have clearly seen that the existence of an inertial frame is guaranteed only when there is no force anymore. So when a body of matter at rest or in uniform motion in a straight line (in an inertial frame) is accelerated then it keeps on accelerating in deep space as long as there is rate of change of momentum or in other words force being constantly applied to the body. Therefore as soon as the force is vanished the rate of change is vanished and the acceleration vanishes and now the same body comes back into an inertial frame but this time it keeps on moving in uniform motion in a straight line with the maximum velocity that it got from previous acceleration. We can conclude that it will never keep on going under acceleration because the force has been removed. So therefore the body will never achieve greater velocity till we apply greater force. There was absolutely no need to put a cosmic speed limit in Newton's first or second law of motion. We could have extended Newton's first law of motion simply as below

"A body continues in its state of uniformly accelerated motion in a straight line as long as the compelling force is acting otherwise."

If Einstein had read the above extension of Newton's first law of motion he would not have mixed up inertial, non-inertial and gravitational.

Inertial and non-inertial motion is possible in straight lines only where as gravitational motion is possible in curves only. The falling of a stone under gravity is the approximation of gravitational motion by using uniformly accelerated non-inertial motion equations of Newton. When the same motion of the falling of a stone is observed from a fixed point in space it is curved and not straight.

In the last equation above, $a$ becomes the centripetal gravitational acceleration $g$ in uniform circular motion and the force become gravitational.

We arrive at the following equation

$$
F=m_{0} a \frac{\left(1+\frac{v^{2}}{c^{2}}\right)}{\left(1-\frac{v^{2}}{c^{2}}\right)^{2}}
$$

Let us analyze the above equation in the light of NR as follows:

$$
\begin{gathered}
F=m_{0} a\left(1+\frac{v^{2}}{c^{2}}\right)\left(1-\frac{v^{2}}{c^{2}}\right)^{-2} \\
F=m_{0} a\left(1+\frac{v^{2}}{c^{2}}\right)\left(1+2 \frac{v^{2}}{c^{2}}+3 \frac{v^{4}}{c^{4}}+4 \frac{v^{6}}{c^{6}}+\ldots \infty\right) \\
F=m_{0} a\left(1+\frac{v^{2}}{c^{2}}\right)+m_{0} a\left(1+\frac{v^{2}}{c^{2}}\right)\left(2 \frac{v^{2}}{c^{2}}+3 \frac{v^{4}}{c^{4}}+4 \frac{v^{6}}{c^{6}}+\ldots \infty\right) \\
F=m_{0} a\left(1+\frac{v^{2}}{c^{2}}\right)+\text { infinite series }
\end{gathered}
$$

Getting rid of the distortion in the form of infinite series as before we see that

$$
F=m_{0} a\left(1+\frac{v^{2}}{c^{2}}\right)
$$

Rewriting equation 20 above as

$$
F=m_{0} a^{\prime}
$$

Where

$$
a^{\prime}=a\left(1+\frac{v^{2}}{c^{2}}\right)
$$

or

$$
g^{\prime}=g\left(1+\frac{v^{2}}{c^{2}}\right)
$$

We see that the slightly modified form of Newton's second law regarding uniform circular motion at constant velocity as given by equation 20 approximates Newton's second law for uniform circular motion at Newtonian velocities as follows:

$$
F=m_{0} a^{\prime} \cong m_{0} a \quad v<<c
$$

Since the gravitational mass is the same as the inertial mass (Einstein's weak principle of equivalence), therefore, a remarkably great conclusion can be drawn from the above equation 23, and is that relative gravitational motion increases gravitational acceleration $\mathrm{g}$ (of both photon and ordinary matter) and hence the Newtonian gravitational force. Equation 23 extends Einstein's strong principle of equivalence i.e. inertial acceleration is equal to gravitational 
acceleration by giving the additional term which arises due to relative gravitational motion. I believe that Newton's gravitational planetary motion is as peculiar and different from Newton's formalism of uniformly accelerated motion as is the speed of light peculiar and different from Newton's uniformly accelerated motion velocities.

Table 1. Important findings by natural relativity.

\begin{tabular}{lcl}
\hline & $\begin{array}{l}\text { Gravitational acceleration \& } \\
\text { inertial acceleration }\end{array}$ & $\begin{array}{l}\text { Gravitational mass, } \\
\text { inertial mass \& rest } \\
\text { mass }\end{array}$ \\
\hline $\begin{array}{l}\text { Ordinary } \\
\text { matter }\end{array}$ & $g^{\prime}=a\left(1+v^{2} / c^{2}\right)$ & $M_{g}=M_{i}=M_{0}$ \\
$\begin{array}{l}\text { Photon } \\
\text { matter }\end{array}$ & $g^{\prime}=g\left(1+v^{2} / c^{2}\right)$ & \\
& $a=0$ & $M_{g}=M_{i}$ \\
\hline
\end{tabular}

I also believe that we should rename Einstein's weak principle of equivalence as strong principle and his strong principle of equivalence as weak one in the light of NR.

\section{Tests of NR}

\subsection{Bending of Ray of Light}

Newton's second law of motion as slightly modified by $\mathrm{NR}$ is given by

$$
F=m_{0} a\left(1+\frac{v^{2}}{c^{2}}\right)
$$

Where

$$
a^{\prime}=a\left(1+\frac{v^{2}}{c^{2}}\right)
$$

or

$$
g^{\prime}=g\left(1+\frac{v^{2}}{c^{2}}\right)
$$

Inserting $v=c$ into the above equation and using this $g^{\prime}$ in place of $g$ into equation 31 we have it in the modified form as

$$
\begin{gathered}
c^{2}=2(1+\varepsilon) g R \\
\varepsilon=\frac{c^{2}}{2 g R}-1 \cong \frac{c^{2}}{2 g R} \quad \varepsilon \gg 1
\end{gathered}
$$

Inserting this value of eccentricity $\varepsilon$ from equation 26 above in equation 45 we have

$$
\begin{gathered}
\delta=\frac{2}{\varepsilon}=\frac{4 g R}{c^{2}}=\frac{4 G M}{R c^{2}} \text { radian } \\
\delta=\frac{2}{\varepsilon}=\frac{4 g R}{c^{2}}=\frac{4 G M}{R c^{2}} \times \frac{360}{2 \pi} \times 3600 \text { arcsec }
\end{gathered}
$$

The following data plugged in equation 27 above gives the bending of ray of light when it passes near the sun to be $G=6.67 \times 10^{-11} \mathrm{~N} \cdot \mathrm{m}^{2} / \mathrm{kg}^{2}$
$M=1.99 \times 10^{30} \mathrm{~kg}$

$R=695,500,000 \mathrm{~m}$

$c=299,792,458 \mathrm{~m} / \mathrm{s}$

$\delta \cong 1.75$ arcsec

The above value is in good agreement with general relativity. According to NR when the photon passes near the sun the gravitational acceleration of the photon increases which in turn increases the gravitational force between them. Therefore the universal force of gravitation becomes double in the case of the Sun and photon interaction due to relative gravitational motion which eventually deflects the photon by double the amount as given by Newton's classical physics.

\subsection{Precession of the Perihelion of Mercury-Distorted \& Actual}

As I said earlier in Article 3 "Analysis of inertial time dilation" last paragraph that at velocities far less than the speed of information NR gives the distorted values which appreciably accounts for the explicable remainder (in astronomical data). Let us check this by calculating the observed/distorted/accurate/relative/apparent/recorded value of the relativistic portion of the precession of the perihelion of Mercury.

Rewriting equation 19 and approximating it to the first order we get

$$
\begin{gathered}
F=m_{0} a\left(1+\frac{v^{2}}{c^{2}}\right)+m_{0} a\left(1+\frac{v^{2}}{c^{2}}\right)\left(2 \frac{v^{2}}{c^{2}}+3 \frac{v^{4}}{c^{4}}+4 \frac{v^{6}}{c^{6}}+\ldots \infty\right) \\
F \cong m_{0} a\left(1+\frac{v^{2}}{c^{2}}\right)+2 m_{0} a \frac{v^{2}}{c^{2}} \cong m_{o} a\left(1+3 \frac{v^{2}}{c^{2}}\right) \\
F \cong m_{0} a\left(1+3 \frac{v^{2}}{c^{2}}\right) \\
a^{\prime} \cong a\left(1+3 \frac{v^{2}}{c^{2}}\right)
\end{gathered}
$$

or

$$
\begin{gathered}
g^{\prime} \cong g\left(1+3 \frac{v^{2}}{c^{2}}\right) \\
g^{\prime} \cong g\left(1+\frac{v^{2}}{c^{2}}+2 \frac{v^{2}}{c^{2}}\right)
\end{gathered}
$$

The term of $2 \frac{v^{2}}{c^{2}}=\frac{v^{2}}{c^{2}}+\frac{v^{2}}{c^{2}}$ above is indicative of the two-fold spacetime dilation effect to the first order of approximation.

Inserting the above value of centripetal acceleration $a^{\prime}=g^{\prime}$ instead of Newtonian $g=G M / r^{2}$ into equation 48 of article 10 "Planetary motion" [7] we have

$$
\frac{\mathrm{d}^{2} u}{\mathrm{~d} \theta^{2}}+u=\frac{G M}{h^{2}}\left(1+3 \frac{v^{2}}{c^{2}}\right)
$$

We know that $h=v r=$ constant, $v=h / r \quad \&$ $u=1 / r$. Putting the value of $v$ into the above equation we have 


$$
-3 \frac{G M}{c^{2}} u^{2}+u+\frac{\mathrm{d}^{2} u}{\mathrm{~d} \theta^{2}}-\frac{G M}{h^{2}}=0
$$

where $P=3 \frac{G M}{c^{2}} \quad \& \quad Q=\frac{G M}{h^{2}}$

$$
-P u^{2}+u+\left(\frac{\mathrm{d}^{2} u}{\mathrm{~d} \theta^{2}}-Q\right)=0
$$

Solving this algebraically for $u$ gives

$$
u=\frac{-1+\sqrt{1+4 P\left(\frac{\mathrm{d}^{2} u}{\mathrm{~d} \theta^{2}}-Q\right)}}{-2 P}
$$

The second term inside the square root is much less than 1so we can consider just the first couple of terms of the expansion, we have

$$
\begin{gathered}
u=\frac{1}{-2 P}\left[-1+\left\{1+4 P\left(\frac{\mathrm{d}^{2} u}{\mathrm{~d} \theta^{2}}-Q\right)\right\}^{1 / 2}\right] \\
\mathrm{u}=\frac{1}{-2 P}\left[-1+1+\frac{1}{2} * 4 \mathrm{P}\left(\frac{\mathrm{d}^{2} u}{\mathrm{~d} \theta^{2}}-Q\right)+\frac{\frac{1}{2} \times\left(\frac{1}{2}-1\right)}{2 !}\left\{4 \mathrm{P}\left(\frac{\mathrm{d}^{2} u}{\mathrm{~d} \theta^{2}}-Q\right)\right\}^{2}+\ldots \infty\right]
\end{gathered}
$$

Simplifying and re-arranging terms, we get

$$
(1+2 \mathrm{PQ}) \frac{\mathrm{d}^{2} u}{\mathrm{~d} \theta^{2}}+u=Q(1+P Q)+P\left(\frac{\mathrm{d}^{2} u}{\mathrm{~d} \theta^{2}}\right)^{2}
$$

The last term on the right hand side of the above equation is negligibly small so we essentially have an equation of the form of equation 49 with

$$
\begin{gathered}
(1+2 \mathrm{PQ}) \frac{\mathrm{d}^{2} u}{\mathrm{~d} \theta^{2}}+u=Q(1+P Q) \\
\Omega=\frac{1}{\sqrt{1+2 P Q}} \cong \frac{1}{1+P Q} \quad \mathrm{p}=\frac{1}{Q(1+P Q)}
\end{gathered}
$$

Hence the distorted Newton-Kepler orbit is described by the relationship below

$$
r(\theta)=\frac{\frac{1}{Q(1+P Q)}}{1+\varepsilon \cos \left(\frac{\theta}{1+P Q}\right)}
$$

The above expression is not the equation of an ellipse but rather it is the equation of a pre-cessing ellipse-like orbit of a planet which is pre-cessing in the direction of increase of radial angular position or motion, because the period of the radial function is not exactly equal to the period of the angular position $\theta$ or in other words the coefficient of $\theta$ is less than unity, in the absence of the tugs of other planets. The angular travel necessary to go from one perigee to the next, for example, is not $2 \pi$ but rather $2 \pi(1+P Q)$. Hence the ellipse precesses/advances by the amount $2 \pi P Q$ radian per revolution. Putting the values of $P \& Q$ from above we have

$$
2 \pi \mathrm{PQ}=2 \pi \times 3 \frac{G M}{c^{2}} \times \frac{G M}{h^{2}}=6 \pi\left(\frac{G M}{h c}\right)^{2} \text { radian per revolution }
$$

This gives the precession in units of radian per revolution of the planet. To convert this to units of arcsec per century for the planet Mercury we note that Mercury completes 414.9 revolutions per century we multiply the above expression by

$$
414.9 \times \frac{360}{2 \pi} \times 3600
$$

So the explicably distorted (measured by astronomical instruments, and hence having the permanent two-fold relativistic spacetime dilation effect, and not the actual/exact) remainder for the relativistic portion of precession of the planet Mercury due to relative motion between the Sun and Mercury in the absence of the tugs/perturbations of other planets in units of arcsec per century is

$$
6 \pi\left(\frac{G M}{h c}\right)^{2} \times 414.9 \times \frac{360}{2 \pi} \times 3600
$$

By substituting the data [7] below we have

$$
\begin{aligned}
& h=v_{\min } r_{\text {max }} \text { (at apogee) } \\
& v_{\text {min }}=38,860 \mathrm{~m} / \mathrm{s} \\
& r_{\text {max }}=69,816,927,000 \mathrm{~m} \\
& c=299,792,458 \mathrm{~m} / \mathrm{s} \\
& G=6.67 \times 10^{-11} \mathrm{~N} \cdot \mathrm{m}^{2} / \mathrm{kg}^{2} \\
& M=1.99 \times 10^{30} \mathrm{~kg} \\
& 6_{\pi}\left(\frac{G M}{h c}\right)^{2} \times 414.9 \times \frac{360}{2 \pi} \times 3600=42.96 \cong 43 \text { arcsec per century }
\end{aligned}
$$

This value by NR is in good agreement with general relativity. Now let us attempt to find the actual value for the relativistic portion of the precession of the planet Mercury due to relative motion between the sun and Mercury in the absence of tugs of other planets. The actual equation of Newton's law of motion in relative motion, after getting rid of distortion (two-fold spacetime dilation effect) caused by the characteristics of the speed of our information in combination with relative motion between the Sun and Mercury and slightly modified by NR, is given by equation 20 above as

$$
\begin{gathered}
F=m_{0} a\left(1+\frac{v^{2}}{c^{2}}\right) \\
a^{\prime}=a\left(1+\frac{v^{2}}{c^{2}}\right)
\end{gathered}
$$

or

$$
g^{\prime}=g\left(1+\frac{v^{2}}{c^{2}}\right)
$$

Inserting the above value of centripetal acceleration $a^{\prime}=g^{\prime}$ instead of Newtonian $g=G M / r^{2}$ into equation 48 of article 10 "Planetary motion" [7] we have

$$
\frac{\mathrm{d}^{2} u}{\mathrm{~d} \theta^{2}}+u=\frac{G M}{h^{2}}\left(1+\frac{v^{2}}{c^{2}}\right)
$$


We know that $h=v r=$ constant, $v=h / r \quad \&$ $u=1 / r$. Putting the value of $v$ into the above equation we have

$$
-\frac{G M}{c^{2}} u^{2}+u+\frac{\mathrm{d}^{2} u}{\mathrm{~d} \theta^{2}}-\frac{G M}{h^{2}}=0
$$

The above equation shows that the only change now is that the value of $P$ is reduced by three times. The rest of the calculations are as before. So we see that this time the precession is reduced by three times. Therefore the actual explicable relativistic precession (which is also a kind of permanent distortion of the Newton-Kepler orbits due to the finite speed of gravitational information in combination with relative motion as will be explained later in article 11 on the "Simple unification of gravitation and electromagnetism" in the form of ellipse-like naturally precessing orbits) of Mercury, which is luckily observed and recognized by astronomical instruments on earth or in space distortedly to be 42.96 arcsec per century by the Astronomers due to the finite speed of light and relative gravitational motion between Mercury and the Sun in the absence of the tugs of other planets, is given by

$$
\frac{1}{3} * 6 \pi\left(\frac{G M}{h c}\right)^{2} \times 414.9 \times \frac{360}{2 \pi} \times 3600=14.32 \text { arcsec per century }
$$

When we look at what the Astronomers have observed to be the total precession rate of the perihelion of Mercury, we see that they advocate a figure of 5600 arc second per century for the total precession of Mercury measured on earth including precession due to our equinox reference line, tugs of other planets and relativistic portion. Since NR predicts the actual/exact value of the relativistic portion of precession of Mercury's perihelion to be 14.32 arc second and an observed/accurate/distorted/apparent remainder value of 42.96 arc second, therefore, we can conclude on the same line of thought, that the actual/exact total precession rate of Mercury is one-third of the total observed/distorted/apparent value of 5600 arc second per century which is around 1867 arc second. This value of 1867 arc second also reinforces and proves the legitimacy of the doubt in the minds of many researchers [7] regarding the values of the portion of the precession of the perihelion of Mercury due to perturbing effects of other planets calculated by using the concept of the planet's mass as being distributed uniformly in a ring around the Sun with a radius equal to the radius of the planet's actual orbit. In contrast, a uniform ring of matter always exerts a net outward pull on an interior planet. In view of this, the representation of the outer planets as uniform rings is certainly questionable and hence spurious.

One may wonder that if the Newtonian model fails in the remainder part of predicting the relativistic precession of Mercury how could it have predicted correctly the bigger part of the precession due to perturbing effects of other planets. Therefore, it was just a mere coincidence that Nature did not find any other way to guide researchers to the explicably observed/accurate/apparent remainder of 43 arc second per century but with a mistake.
When measurements are done with the speed of light in relative motion there stems out a relative value of the quantity. From common sense we know that for the existence of an observed/distorted/relative/accurate/apparent value there must be an actual/exact value behind the curtains. The figure of 1867 arc second is that value. Since the Astronomers have measured the total perihelion precession of Mercury with the help of a finite speed of information in combination with relative motion, therefore, we cannot accept that value of 5600 arc second to be the exact/actual value but we can accept it as the accurate/distorted/relative/apparent value in line with the present theory. So what the previous researchers/astronomers have shown to the world is just an apparent/relative value. However, for the sake of extending the two-body motion Newton's universal law of gravitation, we are not concerned with any value other than the actual/exact frame-independent 14.32 arc second per century.

The laws of Nature are such that they cannot be changed to explain physics or mathematics or our human psychological system but rather the physics and mathematics be got rid of distortions/singularities due to physical immeasurability to explain the laws of Nature.

\subsection{Gravitational Shift in Frequency of Photons.}

We know that light is an electromagnetic wave phenomenon $[2,4]$ and therefore has wave characteristics as well. From Planck's law the energy [1] associated with the wave of a photon (electromagnetic radiation) is $E=h v$. Now considering equation 14 as below

$$
E=m_{0} c^{2}+m_{0} v^{2} \quad 0 \leq v \leq c
$$

Putting the value of the speed for a photon in the above equation we see that it gives us

$$
E=m_{0} c^{2}+m_{0} c^{2}
$$

The first term on the right hand side of the equation above is the reserve energy of the photon. This amount of energy is the reserve energy that the photon has in order to make changes in its wave behavior possible. Because neither the inertial mass of the photon change nor its gravitational mass nor its speed in vacuum. When it falls in a higher gravitational potential it increases its frequency to respond to change in gravitational potential by extracting a very small amount of reserve energy, equal to a certain fraction of that reserve amount of energy. The change in frequency from a lower value to a higher value requires energy according to Planck's law as below

$$
\begin{gathered}
\alpha m_{0} c^{2}=h \Delta v \quad \alpha \leq 1 \\
v_{2}-v_{1}=\alpha \frac{m_{0} c^{2}}{h} \\
\left(v_{2}-v_{1}\right)_{\max }=\frac{m_{0} c^{2}}{h} \quad \alpha=1
\end{gathered}
$$




$$
\begin{gathered}
m_{0} G M\left(\frac{1}{R_{2}}-\frac{1}{R_{1}}\right)=\alpha m_{0} c^{2} \\
\alpha=\frac{G M}{c^{2}}\left(\frac{1}{R_{2}}-\frac{1}{R_{1}}\right) \\
v_{2}-v_{1}=\alpha \frac{m_{0} c^{2}}{h} \\
v_{2}-v_{1}=\frac{G M m_{0}}{h}\left(\frac{1}{R_{2}}-\frac{1}{R_{1}}\right)
\end{gathered}
$$

Where $R_{1} \& R_{2}$ are distances from the center of the earth. It is remarkable to note that the change in frequency does not depend upon the initial frequency of the photon. This is indicative of the fact that neither the inertial mass of the photon nor the gravitational mass nor the speed of the photon change. I believe if researchers are able to observe the small change in the value of the frequency of photons in the laboratory accurately then they would be able to find the exact mass of the photon by using the above formula of natural relativity. The exact mass of the photon remains to be determined precisely.

\subsection{Gravitational Shift in Time of Clocks}

For the understanding of gravitational shift in clock-time we will utilize Newton's pivotal equation 35 . We need to repeat the inertial thought experiment but this time under gravitation, as went before in Article 2 with two inertial clocks. The gravitational thought experiment will go like this. One gravitational clock is orbiting in a satellite around the earth and another gravitational clock is stationary at the surface of the earth. We need to prove that an orbiting gravitational clock in a satellite orbiting the earth in uniform circular motion behaves like an inertial clock when it comes to measuring the time of the stationary gravitational clock at the same radial distance from the earth as that of the moving gravitational clock in the satellite as follows

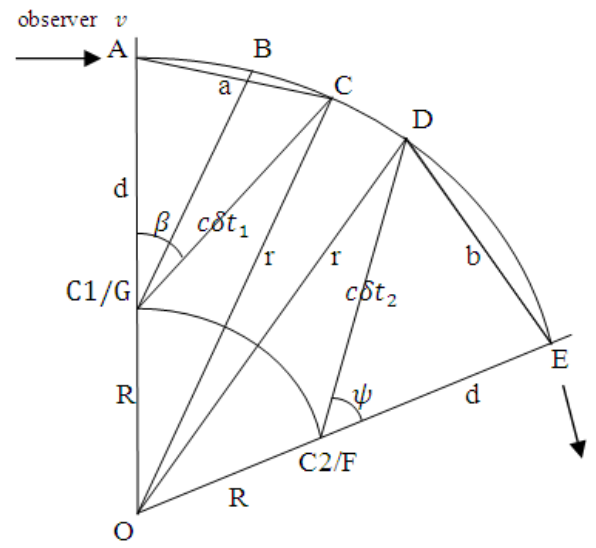

Figure 2. Relative gravitational time dilation. Deriving the time interval $t$ of a clock as a limit with the speed of our information in relative gravitational motion.

Length of $\operatorname{arc} \mathrm{AB}=v t$

Length of arc $\mathrm{BC}=v \delta t_{1}$
Length of arc $\mathrm{BD}=v \delta t_{2}$

Length of $\operatorname{arc} \mathrm{AE}=s$

Length of arc $\mathrm{DE}=s-v T_{2}$

In order to determine the gravitational time dilation of a gravitational clock with respect to distance from the center of the earth I have actually put two stationary clocks (gravitational) on the surface of earth at points $G \& F$, with some spatial curvature apart. I will then calculate the time the moving observer in a satellite measures of the two clocks according to his measurability with another gravitational clock. The equations describing the measurement of time of two different but synchronized clocks in the gravitational rest frame will be scrutinized in the limiting case when the spatial curvature between the two clocks at rest tends to zero, hence making the two different times merge into one observation equal in magnitude. So, while the moving observer moves away from one clock, it is simultaneously moving closer to the second.

In the above Figure 2 an observer is moving from the left to the right in a circular orbit around the earth. Two gravitational clocks $\mathrm{C} 1$ \& $\mathrm{C} 2$ synchronized with each other are placed on the surface of earth some spatial curvature apart. When the observer approaches right on top of the clock $\mathrm{Cl}$ at point $\mathrm{A}$ at a radial distance of $R+d$, the clocks $\mathrm{C} 1 \& \mathrm{C} 2$ start synchronically. After an elapse of time $t$ (according to the moving gravitational clock), the observer reaches point $B$ in its moving frame. Both clocks at rest tick the time of the moving clock $t$ as $t^{\prime}$, just because of difference of gravitational potential, as both are synchronized with each other in the rest frame. Now, the observer tries to get the information of the time of $\mathrm{C} 1$ and by the time $\delta t_{1}$ the information at the speed of light reaches him he has reached point C. Information speed takes definite amount of time (time light or light-like signals take to reach from $\mathrm{C} 1$ to observer at point C). The observer at point $\mathrm{C}$ records this time to be $T_{1}$. Where $\delta t_{1}$ is the definite amount of time in which the information reaches from $\mathrm{C} 1$ to observer at point $\mathrm{C}$.

In the same manner we see that when the same observer gets the information of the time of $\mathrm{C} 2$ he has reached point $\mathrm{D}$. $\mathrm{D}$ does not necessarily need to be on the right of point $\mathrm{C}$, it can be on the left of $\mathrm{C}$ depending upon the prevailing conditions between the observer and the clocks. The same observer records the time of $\mathrm{C} 2$ according to the same power of measurability with which he measured the time of $\mathrm{C} 1$. He measures it $T_{2}$. Where $\delta t_{2}$ is the definite amount of time in which the information reaches from $\mathrm{C} 2$ to observer at point D.

$$
\begin{aligned}
& T_{1}=t+\delta t_{1} \\
& T_{2}=t+\delta t_{2}
\end{aligned}
$$

From $\triangle O C G \& \triangle O D F$ we have, by applying theorem de Al-Kashi, the following equations

$$
\begin{aligned}
& r^{2}=\left(c \delta t_{1}\right)^{2}+R^{2}-2 c \delta t_{1} R \cos (\pi-\beta) \\
& r^{2}=\left(c \delta t_{2}\right)^{2}+R^{2}-2 c \delta t_{2} R \cos (\pi-\psi)
\end{aligned}
$$


Using the above information we obtain

$$
2 t-\left(T_{1}+T_{2}\right)=\frac{2 R}{c}\left\{\frac{\left(t-T_{1}\right) \cos \beta+\left(T_{2}-t\right) \cos \psi}{T_{2}-T_{1}}\right\}
$$

From $\triangle \mathrm{ACG} \& \triangle \mathrm{DEF}$ we have, by applying theorem de Al-Kashi, the following equations

$$
\begin{aligned}
& a^{2}=\left(c \delta t_{1}\right)^{2}+d^{2}-2 c d \delta t_{1} \cos \beta \\
& b^{2}=\left(c \delta t_{2}\right)^{2}+d^{2}-2 c d \delta t_{2} \cos \psi
\end{aligned}
$$

Putting the values of $\delta t_{1} \& \delta t_{2}$ in terms of $T_{1} \& T_{2}$ in the above two equations we have by letting

$$
\begin{gathered}
\omega=\left(t-T_{1}\right) \cos \beta+\left(T_{2}-t\right) \cos \psi \\
2 c d \omega=a^{2}-b^{2}+c^{2}\left\{\left(T_{2}-T_{1}\right)\left(T_{2}+T_{1}\right)-2 t\left(T_{2}-T_{1}\right)\right\}
\end{gathered}
$$

Now using the pseudo sectors ACG and EDF between the concentric circles of radii $R \& R+d$, we have

$$
\begin{gathered}
a \approx v\left(t+\delta t_{1}\right)=v T_{1} \\
b \approx s-v\left(t+\delta t_{2}\right)=s-v T_{2}
\end{gathered}
$$

$\approx$ means that they become equal asymptotically i.e. in the limit

Inserting the values of $a \& b$ into the above equation we obtain

$2 c d \omega=\left(v T_{1}\right)^{2}-\left(s-v T_{2}\right)^{2}+c^{2}\left\{\left(T_{2}-T_{1}\right)\left(T_{2}+T_{1}\right)-2 t\left(T_{2}-T_{1}\right)\right\}$

$\omega=\frac{1}{2 c d}\left[\left(v T_{1}\right)^{2}-\left(s-v T_{2}\right)^{2}+c^{2}\left\{\left(T_{2}-T_{1}\right)\left(T_{2}+T_{1}\right)-2 t\left(T_{2}-T_{1}\right)\right\}\right]$

Rewriting the equation from above as below and inserting the value of $\omega$ from the above equation into the equation below

$$
2 t-\left(T_{1}+T_{2}\right)=\frac{2 R}{c}\left\{\frac{\left(t-T_{1}\right) \cos \beta+\left(T_{2}-t\right) \cos \psi}{T_{2}-T_{1}}\right\}
$$

and applying the limiting case when $s$ tends to zero and the time $T_{2}$ merges to become $T_{1}$ as $T$ asymptotically, we finally obtain

$$
T=\frac{t\left(1+\frac{R}{d}\right)}{\left\{1+\frac{R}{d}\left(1-\frac{v^{2}}{c^{2}}\right)\right\}}
$$

This time, time $T$ is dependent of $d$ and independent of the observer going either to the left or to the right above the clock. This is the equation for relative gravitational time dilation in relative gravitational motion.

If we set the value of $d$ equal to zero in equation $\mathrm{A}$ above then $t$ tends to $t^{\prime}$ because both clocks are at the same gravitational potential (it does not matter one clock is stationary and the other is orbiting in circular orbit, both tick at the same rate as long as they are at the same gravitational potential) and the equation merges to the same equation as equation 4 before of the relative inertial time dilation as below

$$
T=\frac{t^{\prime}}{\left(1-\frac{v^{2}}{c^{2}}\right)}
$$

A remarkable observation can be made by the above fact and is that the gravitational clock, moving in orbit around the earth now near or at the surface of the earth i.e. where $d=0, t$ is equal to $t^{\prime}$, is behaving as a Newton's inertial clock measuring, while in orbital motion, the gravitational time of the stationary gravitational clock, at rest on the surface of the earth, with the speed of our information, as if it were an inertial stationary clock, unaffected by gravity, ticking at rest on the surface of the earth.

$$
T^{\text {ine }}=\frac{t^{g r a}}{\left(1-\frac{v^{2}}{c^{2}}\right)}
$$

or

$$
T_{R}^{i n e}=\frac{t_{R}^{g r a}}{\left(1-\frac{g R}{c^{2}}\right)}
$$

So extending on the same line of thought we can say analogously that at any orbit at $r$ around the gravitating mass/earth the time measured by an orbiting clock of a stationary gravitational clock is related to the gravitational time of the stationary clock, both clocks at the same radial distance from the center of the gravitating mass/earth, by the formula as below

$$
T_{r}^{i n e}=\frac{t_{r}^{g r a}}{\left(1-\frac{g r}{c^{2}}\right)}
$$

where $f_{r}=\frac{1}{\left(1-\frac{g r}{c^{2}}\right)} \& r=R+d$

Another remarkable conclusion that can be made by the above equation is that the fundamental law of reciprocal nature of relativistic time breaks down! The condition of reciprocal nature of relativistic time i.e. both clocks in relativistic motion measure each other's time dilated by the same amount, holds only when both clocks are inertial.

Inertial time is the time measured by an inertial clock which is unaffected by difference in gravitational potential. Therefore a certain interval of time say $t$ measured $T_{r}^{i n e}$ at $r$ is the same as measured $T_{R}^{i n e}$ at $R$ i.e. measured the same but ticked gravitationally (under the influence of gravity) different by two stationary clocks located at $R \& r$.

$$
T_{r}^{\text {ine }}=T_{R}^{\text {ine }}
$$

We can rewrite the above non-reciprocal equations as below 


$$
t_{r}^{g r a}=\left(1-\frac{g r}{c^{2}}\right) T_{r}^{i n e}
$$

and

$$
t_{R}^{g r a}=\left(1-\frac{g R}{c^{2}}\right) T_{R}^{i n e}
$$

Dividing the above equations by each other we have

$$
\frac{t_{r}^{g r a}}{t_{R}^{g r a}}=\frac{\left(1-\frac{g r}{c^{2}}\right)}{\left(1-\frac{g R}{c^{2}}\right)}
$$

or

$$
t_{r}^{g r a}=\frac{\left(1-\frac{g r}{c^{2}}\right)}{\left(1-\frac{g R}{c^{2}}\right)} t_{R}^{g r a}
$$

or

$$
t_{R+d}^{g r a}=\frac{\left(1-\frac{g(R+d)}{c^{2}}\right)}{\left(1-\frac{g R}{c^{2}}\right)} t_{R}^{g r a}
$$

where $r=R+d$

The above equation gives us the shift in time of gravitational clocks placed at two different radial distances from the center of the gravitating mass/earth. The clocks run faster as they go away from matter.

Since equation A above is also non-reciprocal therefore we can write it, after correcting for gravitational time dilation between $t$ and $t^{\prime}$, as below

$$
t_{R}^{g r a}=\frac{\left\{1+\frac{R}{d}\left(1-\frac{v^{2}}{c^{2}}\right)\right\}}{\left(1+\frac{R}{d}\right)} \frac{\left(1-\frac{g R}{c^{2}}\right)}{\left(1-\frac{g(R+d)}{c^{2}}\right)} T_{R+d}^{g r a}
$$

where $v^{2}=g(R+d)$

The dependence on $d$, i.e. radial separation between the clocks causes both the clocks to behave gravitationally. As soon as $\mathrm{d}$ vanishes in the above equation the orbiting clock starts behaving as an inertial stationary clock. The time given by the above equation is the time that a clock on earth will measure, with the speed of our information, of the time of another clock in orbital motion around the earth.

The orbiting clock is neither inertial nor non-inertial but rather it is gravitational and ticks at exactly the same rate as a stationary clock situated at the same orbital radial distance from the center of the earth/gravitating mass as that of the moving one because both are gravitational.

Clocks in aircrafts and planes and those at/near the surface of the earth can be construed as locally inertial and reciprocal for short distances i.e. local effect, and the same formula as given by equation 4 can be used to calculate their relativistic time dilation.

\subsection{Gravitational Shift in the Dimensions of Matter.}

Pursuing on the same line of thought as in the previous Artcle 7.3 we see that the theory developed exposes another remarkable face of gravity i.e. gravity changes the transversal dimension of matter as well along with change in its radial dimension, as predicted by Newton's universal law of gravitation, given below by the similar type of equation as gone before

$$
x_{R+d}^{g r a}=\frac{\left(1-\frac{g(R+d)}{c^{2}}\right)}{\left(1-\frac{g R}{c^{2}}\right)} x_{R}^{g r a}
$$

Let me make it clear at this point in time that this face is also exposed by Newtonian gravity, however never duly understood and appreciated by researches, as shown in the exaggerated Figure 3 below. The transversal component of the radial force of gravity decreases as the body is taken further away from the gravitating mass/earth. So gravity is basically analogous to an artificial fluid whose pressure acts in all directions and increases/decreases in all directions with an increase/decrease in distance from the center of the earth. The change in the gravitational pressure is responded most appreciably by the spring-like highly-elastic spinal vertebrae of the human body of the space travelers by an elongation of couple of centimeters orbiting in space stations around the earth.

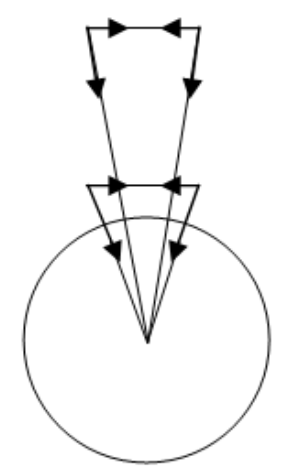

Figure 3. Decrease in the transversal component of the radial force of gravity as the body is taken further away from the gravitating mass.

\section{Orbital Velocity}

According to Newton's theory [2] a body of matter thrown with a velocity tangential to the surface of the earth, the point of projection being at the earth, equal to $v=$ $\sqrt{2 g R}$ where $g$ is the acceleration due to gravity of the earth at the point of projection and $R$ is the radius of Earth, will escape the earth's gravity by making a parabolic path. The range of horizontal tangential velocities greater than $v=\sqrt{g R}$ and less than $v=\sqrt{2 g R}$ will force the object to remain in bounded elliptical orbit around the earth. At a horizontal tangential velocity greater than $v=\sqrt{2 g R}$ the orbit will become hyperbolic. We need an infinite amount of horizontal escape velocity to project a body of matter in an 
exactly horizontal path along a tangential line at the surface of projection. The governing equation from classical Newtonian mechanics for all types of horizontal tangential velocities at a distance $r$, where $\mathrm{r}$ is greater than $R$, from the center of the Earth is the following:

$$
v=\sqrt{(1+\varepsilon) g r}
$$

Where $\varepsilon$ is the eccentricity of the orbit that will be adopted by the projectile. The values $g \& r$ are as mentioned below.

Let us prove equation 31 from the well known polar equation [2] of an orbit (like the orbit of a planet around the Sun) i.e.

$$
r=\frac{h^{2} / k}{(1+\varepsilon \cos \theta)}
$$

Where $h$ is the angular momentum per unit mass of planet around the bigger body and

$$
k=G M=g r^{2}
$$

$G=$ constant of universal gravitation

$M=$ mass of the bigger body i.e. the Sun

$g=$ acceleration due to gravity (as a function of

distance) experienced by the revolving planet

$r=$ radial distance between the center of mass of

the two bodies

The above equation 32 can be reduced to

$$
r=\frac{h^{2} / k}{(1+\varepsilon)}
$$

at the perihelion (the point on the orbit which is closest to the Sun) if we set the value of arbitrary angle $\theta=0^{\circ}$. Now $h=v r$ is the angular momentum per unit mass of the smaller revolving body.

Putting this value of $h$ along with the value of $k$ from equation 33 above, we eventually arrive at

$$
\begin{gathered}
v=\sqrt{(1+\varepsilon) g r} \\
v=\sqrt{g r} \quad \varepsilon=0 ; \quad \text { circular orbit }
\end{gathered}
$$

$\sqrt{g r}<v<\sqrt{2 g r} \quad 0<\varepsilon<1$; elliptical orbit

$v=\sqrt{2 g r} \quad \varepsilon=1$; parabolic orbit

$v>\sqrt{2 g r} \quad \varepsilon>1$; hyperbolic orbit

\section{Hyperbolic Orbit}

Let us find the eccentricity $[5,6]$ in terms of the angle that the two asymptotes (on the right and left of $y$ axis) of the hyperbola make with each other. In order to do so we need to find the slope of the asymptote at infinity. Let point A be the point on the $y$ axis $\mathrm{A}(0, a)$, Point $\mathrm{P}$ on the hyperbola as $\mathrm{P}(x, y)$ and point $\mathrm{B}(x,-a)$ on the horizontal line $y=-a$. Let $\overline{\mathrm{PB}}$ be always the perpendicular distance of the point $\mathrm{P}$ from the Horizontal line $y=-a$ since it's a condition of eccentricity of conic sections. Point $\mathrm{A}$ is fixed and points $\mathrm{P}$ and $\mathrm{B}$ are varying their positions.

Forming the ratio of two lengths

$$
\frac{\overline{\mathrm{AP}}}{\overline{\mathrm{PB}}}=\varepsilon=\frac{\sqrt{(y-a)^{2}+(x-0)^{2}}}{\sqrt{(x-x)^{2}+(y+a)^{2}}}
$$

Rearranging terms we obtain

$y^{2}\left(1-\varepsilon^{2}\right)+x^{2}-2 a y\left(1+\varepsilon^{2}\right)+a^{2}\left(1-\varepsilon^{2}\right)=0$

After completing squares equation 37 can be written as

$y\left(1-\varepsilon^{2}\right)= \pm \sqrt{4 a^{2} \varepsilon^{2}-x^{2}\left(1-\varepsilon^{2}\right)}+\frac{a^{2}\left(1+\varepsilon^{2}\right)^{2}}{\left(1-\varepsilon^{2}\right)}$

If we subtract the term $-\mathrm{a}\left(1+\varepsilon^{2}\right)$ from equation 38 above we get

$y\left(1-\varepsilon^{2}\right)-a\left(1+\varepsilon^{2}\right)= \pm \sqrt{4 a^{2} \varepsilon^{2}-x^{2}\left(1-\varepsilon^{2}\right)}+\frac{a^{2}\left(1+\varepsilon^{2}\right)^{2}-a\left(1-\varepsilon^{4}\right)}{\left(1-\varepsilon^{2}\right)}$

Differentiating equation 37 w.r.t. $x$ and rearranging terms we have

$$
\frac{\mathrm{d} y}{\mathrm{dx}}=\frac{-x}{y\left(1-\varepsilon^{2}\right)-a\left(1+\varepsilon^{2}\right)}
$$

Substituting from equation 39 into equation 40 we have

$$
\begin{gathered}
\frac{\mathrm{d} y}{\mathrm{dx}}=\frac{-x}{ \pm \sqrt{4 a^{2} \varepsilon^{2}-x^{2}\left(1-\varepsilon^{2}\right)}+\frac{a^{2}\left(1+\varepsilon^{2}\right)^{2}-a\left(1-\varepsilon^{4}\right)}{\left(1-\varepsilon^{2}\right)}} \\
\lim _{x \rightarrow \infty} \frac{\mathrm{d} y}{\mathrm{dx}}=\frac{ \pm 1}{\sqrt{\left(\varepsilon^{2}-1\right)}}
\end{gathered}
$$

By equation 42 above the slope of the right asymptote to the hyperbola at infinity is

$$
\frac{1}{\sqrt{\left(\varepsilon^{2}-1\right)}}
$$

If we denote the angle that the asymptote of the hyperbola makes with the $x$-axis by $\alpha$ then the tangent of this angle $\alpha$ is equal to the slope of the asymptote at infinity

$$
\tan \alpha=\frac{1}{\sqrt{\left(\varepsilon^{2}-1\right)}} \cong \frac{1}{\varepsilon} \quad \varepsilon \gg 1
$$

Now $\tan \alpha \cong \alpha$, where $\alpha$ is very small angle in radian

$$
\alpha \cong \frac{1}{\varepsilon}
$$

The angle $\delta$ that the asymptotes (on the left and on the right) make with each other is double the angle $\alpha$ i.e. $\delta=2 \alpha$. This is the deviation from the matter-free straight path of Newton's first law of motion.

$$
\delta \cong \frac{2}{\varepsilon}
$$

\section{Planetary Motion}

For a planet revolving around the Sun [7] at a distance $r$, the Newtonian equations of motion state that the planet has a 
centripetal acceleration of magnitude $G M / r^{2}$ in the direction of the Sun. Since the planet is confined to a single plane, so its position as a function of time can be expressed in terms of the radial magnitude $r(t)$ and an angular position $\theta(t)$ as

$$
x(t)=r(t) \cos \theta(t) \quad y(t)=r(t) \sin \theta(t)
$$

The second derivatives of these coordinates are

$$
\begin{aligned}
& \ddot{x}=\left(\ddot{r}-r \dot{\theta}^{2}\right) \cos \theta-(r \ddot{\theta}+2 \dot{r} \dot{\theta}) \sin \theta=-\frac{G M}{r^{2}} \cos \theta \\
& \ddot{y}=\left(\ddot{r}-r \dot{\theta}^{2}\right) \sin \theta+(r \ddot{\theta}+2 \dot{r} \dot{\theta}) \cos \theta=-\frac{G M}{r^{2}} \sin \theta
\end{aligned}
$$

Since the absolute value of $\theta$ is arbitrary, these equations are equivalent to the conditions obtained by setting the value of the angle equal to 0

$$
\ddot{r}-r \dot{\theta}^{2}=-\frac{G M}{r^{2}} \quad r \ddot{\theta}+2 \dot{r} \dot{\theta}=0
$$

Multiplying the equation on the right above by $r$, we have

$$
r^{2} \ddot{\theta}+2 r \dot{r} \dot{\theta}=\frac{\mathrm{d}}{\mathrm{dt}}\left(r^{2} \dot{\theta}\right)=0
$$

and therefore the quantity in parentheses is constant i.e.

$$
r^{2} \dot{\theta}=h
$$

This represents the conservation of angular momentum and it applies to the central force law. The constancy of this quantity also accounts for Kepler's second law because the incremental area swept out by the position vector in an incremental time is $\mathrm{d} A=(1 / 2) r^{2} \mathrm{~d} \theta$.

Making the substitution $\mathrm{d} \theta / \mathrm{dt}=h / r^{2}$ into the left hand equation of 46 gives

$$
\ddot{r}-\frac{h^{2}}{r^{3}}=-\frac{G M}{r^{2}}
$$

Notice that for a circular orbit all the derivatives of $r$ vanish, and this equation reduces to $h^{2}=r G M$. Making the substitution $h=r^{2} \omega$ where $\omega=\mathrm{d} \theta / \mathrm{dt}$ we have $\omega^{2} r^{3}=G M$, in accordance with Kepler's third law.

We can also use the relation $\mathrm{d} \theta / \mathrm{dt}=h / r^{2}$ to express the derivative of $r$ with respect to time in terms of the derivatives of $r$ with respect to the angular position $\theta$. We have

$$
\begin{gathered}
\dot{r}=\left(\frac{\mathrm{d} \theta}{\mathrm{dt}} \frac{\mathrm{d} t}{\mathrm{~d} \theta}\right) \frac{\mathrm{d} r}{\mathrm{dt}}=\frac{h}{r^{2}} \frac{\mathrm{d} r}{\mathrm{~d} \theta} \\
\ddot{r}=h\left(\frac{\mathrm{d} \theta}{\mathrm{dt}} \frac{\mathrm{d} t}{\mathrm{~d} \theta}\right) \frac{\mathrm{d}}{\mathrm{dt}}\left(r^{-2} \frac{\mathrm{d} r}{\mathrm{~d} \theta}\right)=\frac{h^{2}}{r^{2}} \frac{\mathrm{d}}{\mathrm{d} \theta}\left(r^{-2} \frac{\mathrm{d} r}{\mathrm{~d} \theta}\right)
\end{gathered}
$$

Inserting this expression for the second derivative of $r$ into equation 47 and simplifying gives

$$
\frac{\mathrm{d}}{\mathrm{d} \theta}\left(\frac{1}{r^{2}} \frac{\mathrm{d} r}{\mathrm{~d} \theta}\right)-\frac{1}{r}=-\frac{G M}{h^{2}}
$$

Notice that the quantity in parentheses is just the negative of the derivative of $1 / r$ with respect to $\theta$. Therefore letting $u=1 / r$ we have the simple harmonic equation

$$
\frac{\mathrm{d}^{2} u}{\mathrm{~d} \theta^{2}}+u=\frac{G M}{h^{2}}
$$

In general the solution of an equation of the form

$$
\frac{\mathrm{d}^{2} u}{\mathrm{~d} \theta^{2}}+u=\frac{1}{p}
$$

for constants $\Omega$ and $p$ can be written in the form

$$
u(\theta)=\frac{1}{p}(1+\varepsilon \cos (\Omega \theta))
$$

where $\varepsilon$ is a constant of integration. In the present case we have $\Omega=1$ and $p=h^{2} / G M$. Recalling that $r=1 / u$, the path of the planet in the gravitational field of the Sun of mass $M$ is

$$
r(\theta)=\frac{p}{1+\varepsilon \cos (\Omega \theta)}=\frac{h^{2} / G M}{1+\varepsilon \cos \theta}
$$

If the magnitude of $\varepsilon$ is less than 1 , this is the polar equation of an ellipse with the origin at one focus (Kepler's first law) and with semi-latus rectum $p=h^{2} / G M$.

\section{Simple Unification of Gravitation and Electromagnetism}

Let us reconsider the above equation 23

$$
g^{\prime}=g\left(1+\frac{v^{2}}{c^{2}}\right)
$$

Substituting the value of $g=G M / r^{2}$ and $c=$ $1 / \sqrt{\varepsilon_{o} \mu_{o}}$ into the above equation we see that it looks like the equation below

$$
\begin{gathered}
g^{\prime}=\frac{G M}{r^{2}}\left(1+\varepsilon_{o} \mu_{o} v^{2}\right) \\
F^{\prime}=\frac{G M m}{r^{2}}\left(1+\varepsilon_{o} \mu_{o} v^{2}\right)
\end{gathered}
$$

The above equations contain the two important parameters of gravitation i.e. $G \& g^{\prime}$ and two important parameters of electromagnetism i.e. $\varepsilon_{o} \& \mu_{o}$. This is the proposed simple unification of gravitation and electromagnetism or an extension of Newton's universal law of gravitation. Equation 50a gives the universal law of gravitation (two-body motion) for precessing orbits.

I believe that the part played by electromagnetism is nothing more than a superb natural network of information of Newtonian gravitational force (previously known as force at a distance) in relative gravitational planetary motion in the form of electromagnetic gravitational waves (EGW). These EGWs are, I believe, similar to ordinary electromagnetic waves but pole apart different in their function and propagation. The difference can easily be understood if we compare the ordinary construction steel with post-tensioned strands of steel wires. We see that if the speed of gravita- 
tional information in equation 50a becomes infinite then the equation reduces to Newtonian gravitation i.e. force at a distance (infinite speed of gravitational information).

I believe that the information of the generation/reception of EGWs is clearly indicated by the parameter $M$ in the above equation $50 \mathrm{a}$ i.e. the central body around which the smaller body is rotating. We are familiar with the fact that accelerating charges generate electromagnetic waves and therefore accelerating masses must also do the same. So in the scenario of cosmic catastrophe of the sudden vaporization of the sun, the probability of the earth to permanently keep going on in outer space by making a tangential path to its orbit about 8 minutes after the vanishing of the sun and the coming of the last EGW wave-front to the earth is very low or non-existent. Because as soon as the sun vanishes, the parameter $M$ vanishes in equation 50, but after the lapse of about 8 minutes, instantaneously another $M^{\prime}$ will be ready to take the position in equation 50 above and will reset the generation of EGW's as per routine of the superb natural network of information of Newtonian universal gravitational force. That $M^{\prime}$ will now be the central mass for the earth to rotate or move, about which the sun was once a rotating or moving mass. The speed of information of the EGW will be the same as $c$, as before, but this time the information conveyed to the earth by the EGW of $M^{\prime}$ will be different than what it was prior to the catastrophe. Similarly, for the rest of the planets of the once solar system, a brilliantly new system, as much delicate as the previous solar system, will be developed automatically. This is the surprising backup that, I believe, the nature has in its store for the so called cosmic catastrophe. Conjecturing in line with what I have just said above, we can conclude that our cosmos, and not just our solar system, is under the sway of stable equilibrium even under extreme cases of cosmic catastrophe.

Now once again this speed of gravitational information (EGW) is finite and the same as the speed of an electromagnetic wave in free space, therefore, it must have caused some kind of permanent relativistic distortion somewhere in combination with relative motion. The relativistic distortion caused this time is slightly different since it involves only one term of infinite series which is actually enough, than the ones occurred before and it is the relativistic portion of distortion of the Newton-Kepler orbits in the form of naturally precessing ellipse-like orbits. That's why the planet Mercury precesses (relativistic portion of precession only) by the amount 14.32 arcsec per century every century because it is continuously receiving gravitational information (EGW) from the sun at a finite speed. Newton-Kepler static orbits are an ideal case of receiving gravitational information at an infinite speed (force at a distance). The perpetual planetary relativistic portion of precession tells us that the accelerating masses i.e. the sun or earth (earth is controlling moon motion) are continuously sending EGW.

Moreover, the earth and the sun are not just sending the gravitational information, they are also receiving gravitational information because earth is bound to follow the sun and the sun is bound to move around a black-hole as found by researchers.

So accelerating masses i.e. planets, don't just generate EGW but also receive them. The perpetual relativistic portion of precession of the planets revolving around the sun is basically the permanent distortion caused by the finite speed of gravitational information (EGW) in combination with relative motion. But when we observe this relativistic perpetual precession with the speed of our information it is again measured distortedly to be 42.96 arcsec per century in the case of Mercury. The distance between the sun and the planet or between mercury and the observing space probes or earth does not matter at all. What matters is the finite speed of information in combination with relative planetary gravitational motion approximated in our case with relative Newtonian uniform circular motion. The speeds of information come out to be the same as the speed of an electromagnetic wave as given by Maxwell's equations. When gravitational information speed is involved then precession or advance is more probable to happen than recession as far as orbital motion is concerned.

Let us consider two points $M_{1} \& M_{2}$ in the orbit of Mercury around the sun. Point $M_{1}$ is closer to the perihelion than point $M_{2}$. Suppose the planet Mercury is going towards aphelion. When Mercury passes through point $M_{2}$ it receives the gravitational information of point $M_{1}$ instead of $M_{2}$. The gravitational force at point $M_{1}$ is more than at $M_{2}$ in accordance with equation 50 above. Therefore it experiences the gravitational force of point $M_{1}$ at point $M_{2}$ and hence precesses in the direction of motion due to the delay in receiving the information of gravitation at point $M_{2}$ because of the lack of infinite speed of gravitational information.

\section{Conclusion}

The theory that I have set forth tells us about an amazing reality regarding the relative planetary gravitational motion i.e. the actual relativistic portion of the precession rates of the planets about the Sun i.e. two-body motion which can never ever be measured by the speed of our information but could only be calculated. My theory explains spacetime and the atomic level both. It can also be utilized to find the exact mass of photon which is yet to be determined precisely.

We should avoid mixing inertial relativity with Newton's formalism of uniformly accelerated motion or any of its results but rather we should use Newton's formalism of universal gravitational motion i.e. the case of the uniform circular motion at constant speed.

\section{Acknowledgments}

I would like to thank Campsie Public Library, NSW, Australia, to let me borrow books and use their online system to access internet. This research has been carried out independently. 


\section{References}

[1] Beiser, A, Concepts of Modern Physics (2003)

[2] Adams, R. A., Calculus: A Complete Course (2002)

[3] Red aka, Our Nine Planets and their Specifications, Oct 05 2007
[4] Griffiths, D. J., Introduction to Electrodynamics, $1^{\text {st }}$ Edition

[5] Stewart, J., Calculus (1999), $4^{\text {th }}$ Edition, Brooks/Cole Publishing Company, an ITP Company

[6] Dakin, R. I. Porter, Elementary Analysis (1971)

[7] http://www.mathpages.com/home/kmath280/kmath280.htm. Accessed 3/4/2013. 